\title{
Evaluating Penetration Depth of Treatment Fluids into Dentinal Tubules Using the GentleWave ${ }^{\circledR}$ System
}

Prashanthi Vandrangi*

Sonendo Inc., Laguna Hills, California, USA

*Corresponding author: Dr. Prashanthi Vandrangi, Manager, Sonendo Inc., 26061 Merit Circle, Suite 102, Laguna Hills, CA 92653, USA, Tel: 9095564923; E-mail: shanthi@sonendo.com

Rec date: Feb 01, 2016; Acc date: Feb 25, 2016; Pub date: Mar 02, 2016

Copyright: (C) 2016 Vandrangi P. This is an open-access article distributed under the terms of the Creative Commons Attribution License, which permits unrestricted use, distribution, and reproduction in any medium, provided the original author and source are credited.

\begin{abstract}
This study evaluates the depth of sodium hypochlorite $(\mathrm{NaOCl})$ penetration into dentinal tubules by using the GentleWave ${ }^{\circledR}$ System versus ultrasonic agitation. Forty extracted human molars were accessed and instrumented to size \#15 with taper .04 , cleaned to remove pulp tissue, immersed in crystal violet dye, and incubated $\left(37^{\circ} \mathrm{C}\right)$ overnight. Samples were rinsed with distilled water for 30 minutes and randomly divided into four treatment groups with $\mathrm{NaOCl}$ as the treatment fluid ( $\mathrm{n}=10$ molars each): (1) controls (no treatment), (2) passive ultrasonic activation using PiezonMaster ${ }^{T M} 700$ (EMS) with ESI-tip, (3) active ultrasonic activation using PiezonMaster 700 with ESI-tip with maximum irrigation rate, and (4) the GentleWave System. Following the treatments, samples were rinsed with distilled water for one minute. Crowns were removed and roots were carefully split longitudinally, of which only $70 \%$ roots were free of cutting artifacts. Mesiobuccals and distobuccals from maxillary molars and mesials from mandibular molars (74 root halves) were evaluated for this study. The depth of $\mathrm{NaOCl}$ penetration into dentinal tubules was imaged and analyzed using Nikon ${ }^{\circledR}$ stereo-microscope and software. Statistical comparison was done with Welch's t-test $(p<0.05)$. Pearson correlation coefficients $(r)$ were calculated for degree of dependence between depth of $\mathrm{NaOCl}$ penetration and distance from the apex. Group 4 when compared to Group 3 and Group 2 was significantly different $(p<0.05)$ for the apical region of the root canals. No significant difference was observed between Group 2 and Group 3 ( $p>0.05$ ). In summary, the GentleWave System demonstrated approximately four times greater $\mathrm{NaOCl}$ penetration depth in apical region than active ultrasonic system and was effective throughout the root canal system.
\end{abstract}

Keywords: $\mathrm{NaOCl}$; GentleWave ${ }^{\oplus}$ System; Multisonics ${ }^{\mathrm{Tw}}$ energy; Molars; Root canal therapy

\section{Introduction}

The goal of endodontic treatment is to eliminate microbial infection from the root canal system to allow healing of apical periodontitis [1]. Studies have shown that bacteria can invade not only the main root canal system but also into the dentinal tubules [1,2]. The extent of endotoxin penetration can be as high as $500 \mu \mathrm{m}$ with intact cementum [3].

In order to eradicate bacteria and remove tissue debris, current protocols rely on instrumentation and effective irrigation [4]. Irrigation plays an important role in the disinfection of the root canal system. Sodium hypochlorite $(\mathrm{NaOCl})$ is the most widely used treatment fluid because of its ability to dissolve pulp tissue, kill microbes, and detach biofilms [5-8]. The concentration, temperature, and contact time, and the mode of delivery of $\mathrm{NaOCl}$ are important determinants of its effectiveness during root canal treatments [9-11]. Many technologies, such as ultrasonics, have been employed in the clinic to maximally facilitate the effect of irrigation.

Several studies have shown that agitation of treatment fluids is directly associated with the amount of tissue debris being removed from the main root canal system $[10,11]$. However, very little information is available about the penetration depth of $\mathrm{NaOCl}$ into dentinal tubules using various endodontic devices $[12,13]$. In an in vitro study using crystal violet stained dentin, $\mathrm{NaOCl}$ penetration depths between 77 and $300 \mu \mathrm{m}$, depending on the concentration, temperature, and time of exposure of $\mathrm{NaOCl}$ [12]. The deepest penetration of $300 \mu \mathrm{m}$ was measured at $20 \mathrm{~min}$ by $6 \% \mathrm{NaOCl}$ heated to $45^{\circ} \mathrm{C}$. Wong and Cheung utilized a dual-species film (Enterococcus faecalis and Porphyromonas gingivalis) and showed that $3 \% \mathrm{NaOCl}$ showed some effectiveness for up to $200-300 \mu \mathrm{m}$ into dentin [13]. The effect of agitation on penetration depth, e.g. by sonic or ultrasonic devices has not been studied.

A novel endodontic system, the GentleWave ${ }^{\oplus}$ System (Sonendo, Laguna Hills, CA), delivers treatment fluids non-invasively to root canals by utilizing a combination of acoustics and advanced fluid mechanics [14]. A high speed, degassed treatment fluid is delivered into the pulp chamber of the tooth by a Treatment Instrument ${ }^{\text {tax }}$ positioned on the occlusal surface of an accessed tooth. The treatment fluid flow reaches the entire root canal system while a built-in suction within the treatment instrument removes the excess fluid $[15,16]$. Since the GentleWave System has been shown to greatly remove tissue debris [16], it is of interest to study the penetration into the dentinal tubules of $\mathrm{NaOCl}$ agitated by the GentleWave System in comparison to ultrasonic agitation.

\section{Materials and Methods}

\section{Ethics statement}

The teeth used for this study were indicated for extraction for other purposes, either periodontal reasons or for decay. Once indicated for 
extraction and after extraction, deidentified human first or second molars (both mandibular and maxillary) were collected from independent clinicians and stored in phosphate buffered saline solution (PBS) at $4^{\circ} \mathrm{C}$ until use. Patients were informed about the research purposes and gave verbal informed consent, which was not recorded to keep the procedure anonymous.

\section{Sample selection}

Teeth were radiographically assessed and only teeth that met the study inclusion criteria were utilized. Any teeth with decay or fractures below the cemento-enamel junction (CEJ), internal or external root resorption, open apices, or previous root canal therapy were excluded. A total of 40 teeth were utilized for the study.

\section{Sample preparation}

When present, caries were removed and missing coronal tooth structure was restored using etchant (Etch-Rite, Pulpdent, Watertown, MA), bonding agent (Optibond, Kerr, Orange, CA), and Virtuoso ${ }^{\circ}$ flowable light-cure composite (Denmat, Lompoc, CA). Following endodontic access, all teeth were firmly secured and sealed within a water-saturated porous medium using an adhesive (McMaster-Carr, Los Angeles, CA) to simulate blood-saturated periapical tissue. Reproducible glide paths and working length were established using a \#10 K-file (MANI K-files, Utsunomiya, Japan) and canals were instrumented with \#15 K-files (MANI K-files, Utsunomiya, Japan) and \#15/.04 EndoSequence rotary files (Brasseler, Savannah, GA). $1 \mathrm{ml}$ of saline was delivered between each file using a syringe and 30G Max-iprobe $^{\otimes}$ needle to flush the dentin debris created during instrumentation. All samples were cleaned using the GentleWave System to remove tissue debris and to establish a baseline [16]. The samples were immersed in crystal violet dye (Fisher Chemical, Waltham, MA) for staining, placed in plastic vials, and incubated at $37^{\circ} \mathrm{C}$ overnight. Samples were then removed from the vials and rinsed under tap water for 30 minutes [3].

\section{Treatment groups}

The teeth were divided into four different treatment groups ( $\mathrm{n}=10$ per group): 1) negative control group (untreated); 2) passive ultrasonic activation with PiezonMaster 700 (EMS) with ESI tip; 3) active ultrasonic activation with PiezonMaster 700 (EMS) with ESI tip with maximum irrigation rate; and 4) the GentleWave System.

\section{Group 1: Negative controls}

Teeth in the control group did not undergo any endodontic treatment after immersion in crystal violet and were used to establish baseline penetration values and confirm penetration of crystal violet dye into the dentinal tubules.

\section{Group 2: Passive PiezonMaster 700 treatment}

Ultrasonic activation was performed using PiezonMaster 700 with a \#15/.02 ESI tip (DT-011, Electro Medical Systems, Nyon, Switzerland) using Endo mode set to maximum power. The tip was placed $4 \mathrm{~mm}$ above the working length. $1 \mathrm{ml}$ of $3 \% \mathrm{NaOCl}$ was injected into each canal using a 30G Maxi-Probe needle. Each canal was then activated three times for 20 seconds for a total time of 5 minutes per tooth [17-21]. $1 \mathrm{ml}$ of distilled water was delivered into each canal using a syringe and 30G Max-i-probe needle to flush any residual $\mathrm{NaOCl}$.

\section{Group 3: Active PiezonMaster 700 treatment}

Ultrasonic activation with irrigation was performed with PiezonMaster 700 with a \#15/.02 ESI tip using Endo mode set to maximum power. The tip was placed $4 \mathrm{~mm}$ above the working length. The handpiece was set to maximum irrigation using $3 \% \mathrm{NaOCl}$ as the treatment fluid. Each canal was activated and irrigated three times for 20 seconds for a total time of 5 minutes per tooth [17-21]. $1 \mathrm{ml}$ of distilled water was delivered into each canal using a syringe and $30 \mathrm{G}$ Max-i-probe needle to flush any residual $\mathrm{NaOCl}$.

\section{Group 4: GentleWave system treatment}

The GentleWave System was used with $3 \% \mathrm{NaOCl}$ for 5 minutes per tooth and distilled water for 15 seconds. The treatment instrument was placed on an accessed occlusal surface to deliver the treatment fluid into the pulp chamber [14-16]. All the canals were treated simultaneously.

\section{Sample processing}

Following treatments, the teeth were cleaned with an air/water syringe for 20 seconds to remove residual $\mathrm{NaOCl}$. The crowns were removed and the roots were carefully split along the longitudinal axis to expose the entire extent of the root canal using a diamond disc (NTI, Rotary Dental Instruments, Kahla, Germany). Unfortunately, some roots were not considered for further evaluation as unintentional artifacts were introduced during splitting. Also, for these studies, only roots with more complex anatomies, namely, mesiobuccals and distobuccal canals from maxillary molars and mesial canals from mandibular molars were evaluated [18,22].

\section{Image acquisition and data analysis}

Root halves free of cutting and splitting artifacts for all groups were examined and imaged with a stereo microscope (Nikon Eclipse-Ci, Nikon, Melville, New York, USA) at both $40 \times$ and $100 \times$ magnifications. The penetration depth of $\mathrm{NaOCl}$ into the dentinal tubules was analyzed using Nikon Elements software. Images were taken on both halves of the root canal at $1 \mathrm{~mm}$ intervals for a total of $9 \mathrm{~mm}$ starting at the apex of the tooth and moving toward the coronal region. The images were grouped for every $3 \mathrm{~mm}$ and will be referred to as apical (1-3 mm), middle (4-6 mm), and coronal (7-9 mm) regions of the canal.

The depth of $\mathrm{NaOCl}$ penetration was analyzed using horizontal line intensity profiles from the root canal toward the periphery, where the depth of $\mathrm{NaOCl}$ penetration was defined as the region where crystal violet dye was removed and a white line was observed within the dentinal tubules. The white line represents the area where $\mathrm{NaOCl}$ "bleached" the dye and penetrated into the tubules. The line intensity profile directly corresponds to the difference in color along the horizontal axis of the image, therefore detecting intensity peaks where the "white" pixels are present. Images from all specimens were evaluated by two blinded operators.

\section{Statistical analysis}

The mean penetration depths were compared using a Welch's t-test (Group 4 versus Group 3, Group 4 versus Group 2, and Group 2 versus Group 3). The results indicated that the residuals were normally distributed, and uniformity was checked by plotting against predicted values; thus, none of the analysis of assumptions was violated. 
Differences in mean penetration depths were considered statistically significant if the p-value was less than 0.05 .

\section{Results}

74 root canals $(28$ mesial canals of mandibular molars, 24 mesiobuccal canals of maxillary molars, and 22 distobuccal canals of maxillary molars) were analyzed for Group 1, Group 2, Group 3, and Group 4. In summary, 15 mandibular molars and 17 maxillary molars survived the splitting process. Penetration of $\mathrm{NaOCl}$ into dentin was detected as a bleached zone from the root canal toward the periphery as shown in Figure 1 and evaluated with microscopy. The effectiveness of three different treatment groups was evaluated in different regions of the root canal system. The values of penetration depth are shown in Figure 2 .

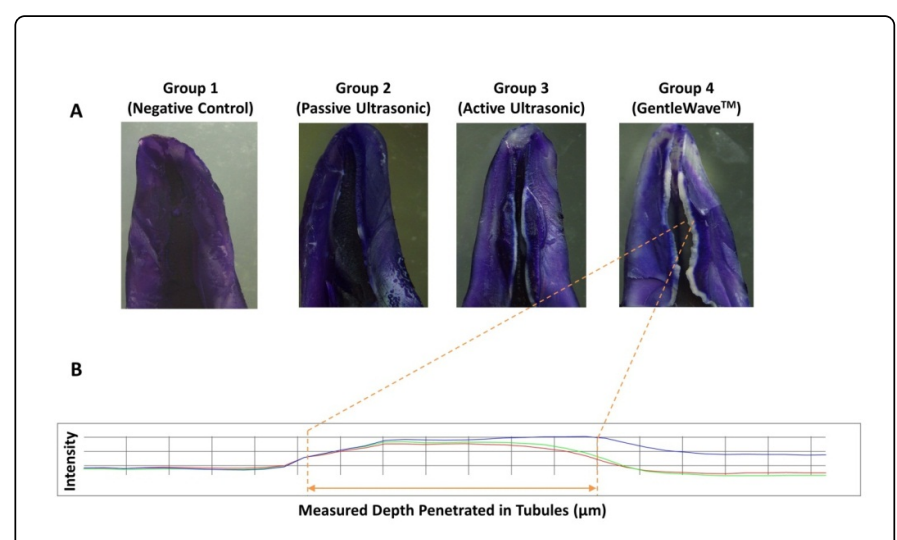

Figure 1: The figure shows representative images of longitudinally split molars dyed with crystal violet. (A) Molars used for negative control (Group 1) and those treated with passive ultrasonic activation (Group 2), active ultrasonic activation (Group 3), and the GentleWave System (group 4). (B) Representative plot of penetration depth of the 'bleached' area.

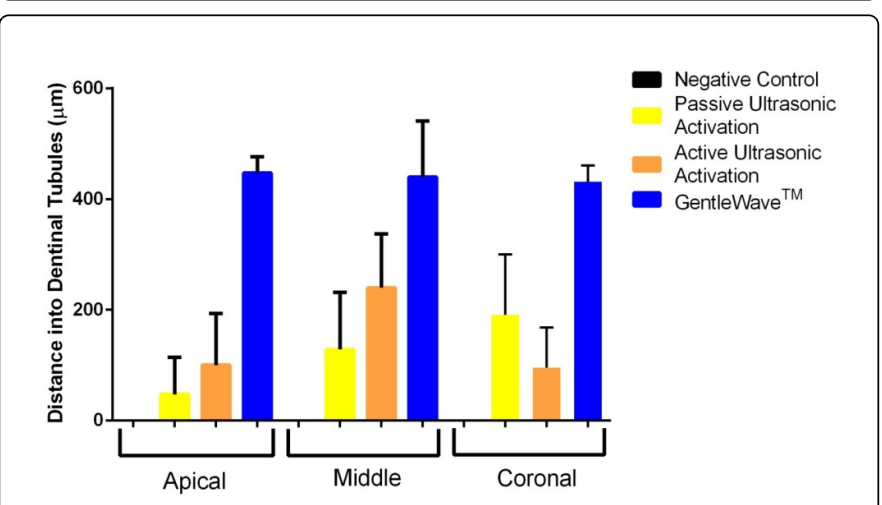

Figure 2: The average penetration depth of $\mathrm{NaOCl}$ into dentinal tubules was measured in crystal violet dyed mesials for Group 1, Group 2, Group 3, and Group 4 (Standard deviation shown by bars). The samples treated in Group 4 not only demonstrate a deeper penetration of $\mathrm{NaOCl}$ but also demonstrate a uniform penetration depth throughout the apical, middle, and coronal regions of the root.
The average depth (average \pm standard deviation) penetrated in Group 4 was $430.9 \pm 30.0 \mu \mathrm{m}$, Group 3 was $209.5 \pm 94.3 \mu \mathrm{m}$, and Group 2 was $112.3 \pm 65.4 \mu \mathrm{m}$ in the coronal region. However, in the middle regions for Group 4, Group 3, and Group 2, the average depth penetration was $439.5 \pm 101.46 \mu \mathrm{m}, 212.2 \pm 96.6 \mu \mathrm{m}, 130.7 \pm 72.7 \mu \mathrm{m}$ respectively and in the apical region, it ranged from $461.3 \pm 59.5 \mu \mathrm{m}$, $129.0 \pm 102.3 \mu \mathrm{m}, 54.1 \pm 78.7 \mu \mathrm{m}$ respectively. The average depth in Group 1 (controls) was $0 \mu \mathrm{m}$. Further, in the coronal region, the shallowest (non-zero) and deepest penetrations were $70.3 \mu \mathrm{m}$ (Group 2) and $494.5 \mu \mathrm{m}$ (Group 4) respectively; in the middle region, the shallowest (non-zero) and deepest penetrations were $39.7 \mu \mathrm{m}$ (Group 2) and $586.4 \mu \mathrm{m}$ (Group 4) respectively; and in the apical region, the shallowest and deepest penetrations were $55.6 \mu \mathrm{m}$ (Group 2) and 521.0 $\mu \mathrm{m}$ (Group 4) respectively.

Group 4 when compared to Group 2 and Group 3 was significantly different $(p<0.05)$ for the apical, middle, and coronal regions of the canals. No significant difference was observed between Group 2 and Group 3 ( $\mathrm{p}>0.05)$.

\section{Discussion}

The methodology used for assessing the penetration of $\mathrm{NaOCl}$ solutions into dentin was adapted from the stained dentin block model developed previously [5]. It has been previously shown that mechanical instrumentation reduces the presence of bacteria from human root canals by approximately $50 \%$ [11]. In addition to mechanical instrumentation, disinfecting fluids are needed to eliminate the microbiota in locations where instruments cannot access the anatomical complexities [23-26]. Even though $\mathrm{NaOCl}$ is an effective disinfectant when in direct contact with biofilm, its cleaning efficiency has been demonstrated only in the coronal and middle thirds but not in the apical third of the canal [27]. Further, studies have demonstrated that although bacteria in smear layers and deeper layers of dentine could be eliminated by procedures such as ultrasonic irrigation with $\mathrm{NaOCl}$ [28], microorganisms within apical third, fins, and isthmi could still be relentless [29]. Even though, bacterial penetration to about $300 \mu \mathrm{m}$ deep into the dentinal tubules has been shown previously, chemical signals namely endotoxins from bacteria, can penetrate approximately 300-500 $\mu \mathrm{m}$ into dentinal tubules [4-6]. This infected or contaminated dentin might serve as a potential source of persistent apical periodontitis.

In the current study, the average depth penetration achieved using GentleWave System was $447.4 \pm 76.5 \mu \mathrm{m}$. These results show that the much needed $500 \mu \mathrm{m}$ penetration depth may be achieved using the GentleWave System. Previous studies have shown the enhanced tissue dissolution rate and removal of the GentleWave, even from the apical third region of the root canal system [14-16]. The GentleWave System cleans the dentinal tubules in the apical region at least 4 times and 8.5 times deeper than the active and passive ultrasonic system, respectively.

It is interesting to note that there was no statistical difference in the penetration depth in the dentinal tubules in apical, middle, and coronal regions when the molars were cleaned with the GentleWave System. However, when cleaned with the active ultrasonic system, we observed that the penetration depth in both the coronal and middle regions of the root canal system was similar, as this technique relies on the transmission of acoustic energy from an oscillating file to the $\mathrm{NaOCl}$ in the root canal space. Also, as the fluid has to propagate to the narrower anatomical area of the apical region of the root canal, 
efficient penetration of $\mathrm{NaOCl}$ was prevented. Further, the tip of the ultrasonic system is likely to be restricted in the apical third, which dampens the efficiency of such devices. Further, the penetration depth using the passive ultrasonic system is less as it does not utilize continuous refreshment of fluid.

On the other hand, the technology of the GentleWave System employs various phenomena including a strong hydrodynamic cavitation cloud which is used to generate a broad spectrum of sound waves (Multisonic ${ }^{\mathrm{ma}}$ technology) within the degassed treatment fluid inside the canal. The degassed treatment fluid contains a reduced amount of dissolved gas to optimize the interplay of the propagating multisonic energy and fluid dynamics. Multisonic energy travels through the fluid into the entire root canal system, hence cleaning the root canal system. Existence of multisonic energy enables effective penetration of waves into micron sized tubules [30].

The temperature in the root canal increases to a maximum of $45^{\circ} \mathrm{C}$, $29^{\circ} \mathrm{C}$, and $40^{\circ} \mathrm{C}$, when the teeth were treated with passive ultrasonic system, active ultrasonic system, and the GentleWave System, respectively $[14,31]$. It has been previously shown that the temperature in the root canal system is always lower than that measured at the external root surface and is dependent on the thickness of the dentin wall [31]. Of particular importance is that the critical level of temperature at the external root surface that does not cause irreversible consequences is $47^{\circ} \mathrm{C}$ [32]. Within the limitations of this study, the three tested modalities do not exceed the critical level of temperature.

A limitation of the present study is that the flow rates of the GentleWave System and the ultrasonic systems are different. The GentleWave System results in a flow rate of $45 \mathrm{ml} / \mathrm{min}$, whereas the ultrasonic systems were set to $15 \mathrm{ml} / \mathrm{min}$ to maximum power in 'endo' modes. However, the flow rate for ultrasonic system was maintained at $15 \mathrm{ml} / \mathrm{min}$ for clinical relevance [14].

Another limitation of the current study is the extent of shaping of the root canals. This prevented any bias that may occur as a result of using different file sizes. In order to accomplish using one standardized size, care was taken to shape all the canals to \#15/.04. For the ultrasonic systems, a tip of $\# 15 / .02$ was used, in order to ensure the vibration of the ultrasonic tip. On the other hand, for the GentleWave System, the tip of the treatment instrument entered only the pulp chamber.

Future studies should probe into the type of dentin and the corresponding depth of penetration of $\mathrm{NaOCl}$ as the later may be dependent on the thickness of the dentin $[2,5,33]$. Future work should also include the comparison of GentleWave with other sonic devices. Further, since crystal violet dye was used as a surrogate to bacteria, it will be interesting to study the cleaning efficiency of GentleWave System on microbial flora.

\section{Conclusion}

In conclusion, within the limitations of this study, the GentleWave ${ }^{\infty}$ System demonstrated at least four times deeper cleaning in the apical region than currently employed ultrasonic systems. The depth of cleaning was independent of the location within the root canal system. Even though further in vitro and in vivo studies are warranted, these results may have clinical implications in the success of root canal treatments.

\section{Acknowledgments}

This study was supported by Sonendo ${ }^{\circ}$. We thank Dr. Markus Haapasalo from the University of British Columbia for his insight. We appreciate Dr. Ronald Perry from Tufts University for his earlier involvement in a pilot study for an undergraduate project.

\section{References}

1. Baumgartner JC, Mader CL (1987) A scanning electron microscopic evaluation of four root canal irrigation regimens. J Endod 13: 147-157.

2. Zehnder M (2006) Root canal irrigants. J Endod 32: 389-398.

3. Horiba N, Maekawa Y, Matsumoto T, Nakamura H (1990) A study of the distribution of endotoxin in the dentinal wall of infected root canals. J Endod 16: 331-334.

4. Haapasalo M, Endal U, Zandi H, Coil JM (2005) Eradication of endodontic infection by instrumentation and irrigation solutions. Endod Topics 10: 77-102.

5. Naenni N, Thoma K, Zehnder M (2004) Soft tissue dissolution capacity of currently used and potential endodontic irrigants. J Endod 30: 785-787.

6. Spratt DA, Pratten J, Wilson M, Gulabivala K (2001) An in vitro evaluation of the antimicrobial efficacy of irrigants on biofilms of root canal isolates. Int Endod J 34: 300-307.

7. Clegg MS, Vertucci FJ, Walker C, Belanger M, Britto LR (2006) The effect of exposure to irrigant solutions on apical dentin biofilms in vitro. J Endod 32: 434-437.

8. Dunavant TR, Regan JD, Glickman GN, Solomon ES, Honeyman AL (2006) Comparative evaluation of endodontic irrigants against Enterococcus faecalis biofilms. J Endod 32: 527-531.

9. Moorer WR, Wesselink PR (1982) Factors promoting the tissue dissolving capability of sodium hypochlorite. Int Endod J 15: 187-196.

10. Baumgartner JC, Cuenin PR (1992) Efficacy of several concentrations of sodium hypochlorite for root canal irrigation. J Endod 18: 605-612.

11. Gu LS, Kim JR, Ling J, Choi KK, Pashley DH, et al. (2009) Review of contemporary irrigant agitation techniques and devices. J Endod 35: 791-804.

12. Zou L, Shen Y, Li W, Haapasalo M (2010) Penetration of sodium hypochlorite into dentin. J Endod 36: 793-796.

13. Wong DT, Cheung GS (2014) Extension of bactericidal effect of sodium hypochlorite into dentinal tubules. J Endod 40: 825-829.

14. Haapasalo M, Wang Z, Shen Y, Curtis A, Patel P, et al. (2014) Tissue dissolution by a novel multisonic ultracleaning system and sodium hypochlorite. J Endod 40: 1178-1181.

15. Ma J, Shen Y, Yang Y, Gao Y, Wan P, et al. (2015) In vitro study of calcium hydroxide removal from mandibular molar root canals. J Endod 41: 553-558.

16. Molina B, Glickman G, Vandrangi P, Khakpour M (2015) Evaluation of root canal debridement of human molars using the GentleWave system. J Endod 41: 1701-1705.

17. Paragliola R, Franco V, Fabiani C, Mazzoni A, Nato F, et al. (2010) Final rinse optimization: influence of different agitation protocols. J Endod 36: 282-285.

18. Yoo YJ, Lee WC, Kim HC, Shon WJ, Baek SH (2013) Multivariate analysis of the cleaning efficacy of different final irrigation techniques in the canal and isthmus of mandibular posterior teeth. Restor Dent Endod 38: 154-159.

19. Böttcher DE, Rahde NM, Grecca FS (2012) Calcium hydroxide removal: effectiveness of ultrasonic and manual techniques. Revista Odonto Ciência 27: 152-155.

20. Wiseman A, Cox TC, Paranjpe A, Flake NF, Cohenca N, et al. (2011) Efficacy of sonic and ultrasonic activation for removal of calcium hydroxide from mesial canals of mandibular molars: a microtomographic study. J Endod 37: 235-238. 
Citation: Vandrangi P (2016) Evaluating Penetration Depth of Treatment Fluids into Dentinal Tubules Using the GentleWave ${ }^{\circledR}$ System. Dentistry 6: 366. doi:10.4172/2161-1122.1000366

Page 5 of 5

21. Braga NM, Brito M Jr, da Silva JM, Miranda LS, de Carvalho JR Jr, et al. (2013) Different ultrasonic vibration protocols and their effects on retention of post-and-core to root canal. Gen Dent 61: 40-42.

22. Garg N and Garg A (2010) Textbook of Endodontics. (1st rev. edn), Boydell \& Brewer Ltd, Suffolk.

23. Dalton BC, Orstavik D, Phillips C, Pettiette M, Trope M (1998) Bacterial reduction with nickel titanium instrumentation. Int Endod J 24: 763-767.

24. Ciucchi B, Khettabi M, Holz J (1989) The effectiveness of different endodontic irrigation procedures on the removal of the smear layer: a scanning electron microscopic study. Int Endod J 22: 21-28.

25. Zehnder M, Schmidlin P, Sener B, Waltimo T (2005) Chelation in roo canal therapy reconsidered. J Endod 31: 817-820.

26. Foschi F, Nucci C, Montebugnoli L, Marchionni S, Breschi L, et al. (2004) SEM evaluation of canal wall dentine following use of MTwo and ProTaper NiTi rotary instruments. Int Endod J 37: 832-839.

27. Cruz EV, Kota K, Haque J, Iwaku M, Hoshino E (2002) Penetration of propylene glycol into dentine. Int Endod J 35: 330-336.
28. Ng YL, Mann V, Rahbaran S, Lewsey J, Gulabivala K (2008) Outcome of primary root canal treatment: systematic review of the literature - Part 2. Influence of clinical factors. Int Endod J 40: 6-31.

29. Peters OA (2004) Current challenges and concepts in the preparation of root canal systems: a review. J Endod 30: 559-567.

30. Charara K, Friedman S, Sherman A, Kishen A, Malkhassian G, et al (2016) Assessment of apical extrusion during root canal procedure with the novel GentleWave system in a simulated apical environment. J Endod 42: 135-139.

31. Cameron JA (1988) The effect of ultrasonic endodontics on the temperature of the root canal wall. J Endod 14: 554-559.

32. Eriksson AR, Albrektsson T (1983) Temperature threshold levels for heat induced bone tissue injury: a vital microscopic study in the rabbit. J Prosthet Dent 50: 101-107.

33. Sarbinoff JA, O'Leary TJ, Miller CH (1983) The comparative effectiveness of various agents in detoxifying diseased root surfaces. J Periodontol 54: 77-80. 